\title{
ALGAS EPIBIONTES EN BRAQUIUROS (CRUSTACEA) DE DOS POCETAS INTERMAREALES EN LA COSTA SURORIENTAL DE CUBA
}

\author{
Algae epibionts in brachyuran (Crustacea) of two intertidal tide pools \\ in the south-east coast of Cuba
}

\author{
Asiel Cabrera Guerrero y Abdiel Jover Capote
}

Grupo Científico Estudiantil de Ecología Marina “Dra. María Elena Ibarra Martín” (EcoMar). Departamento de Biología y Geografía, Facultad de Ciencias Naturales y Exactas, Universidad de Oriente. Patricio Lumumba s/n, Santiago de Cuba, Cuba. CP: 90 500. asiel.cabrera@estudiantes.uo.edu.cu y ajover@uo.edu.cu.

\section{RESUMEN}

Se identifican las algas epibióticas en tres braquiuros que habitan en pocetas intermareales. Los braquiuros se recolectaron mediante buceo SCUBA y se le retiraron todas las algas presentes en el exoesqueleto. Se identifican 15 algas epibióticas sobre Macrocoeloma trispinosum (Latreille, 1825), Omalacantha bicornuta (Latreille, 1825) y Actaea bifrons Rathbun, 1898. Las especies más frecuentes son algas filamentosas y pequeñas de las familias Ceramiaceae y Cladophoraceae. Las algas Sphacelaria tribuloides y Cladophora catenate se identificaron sobre los exoesqueletos de las tres especies de braquiuros.

Palabras clave: enmascaramiento, cangrejos decoradores, interacciones alga-cangrejos.

\section{ABSTRACT}

Epibiotic algae are identified in three brachyuran crabs that inhabit intertidal pools. Crustaceans were collected by SCUBA diving and all the algae present in the exoskeleton were removed. Fifteen epibiotic algae are identified on Macrocoeloma trispinosum (Latreille, 1825), Omalacantha bicornuta (Latreille, 1825) and Actaea bifrons Rathbun, 1898. The most frequent species are filamentous and small algae of the families Ceramiaceae and Cladophoraceae. The algae Sphacelaria tribuloides and Cladophora catenate were identified on the exoskeletons of the three species of brachyrans.

Keywords: masking, Decorator crabs, algae-crab's interactions.

\section{INTRODUCCIÓN}

Las superficies sumergidas de los ambientes marinos están expuestas a la colonización por una gran variedad de organismos presentes en la columna de agua (Schärer, 2005). En el bentos marino es frecuente que se establezcan relaciones no simbióticas entre los organismos sésiles epibiontes que se asientan sobre organismos vivos basibiontes. Estas relaciones son definidas como epibiosis (Taylor y Wilson, 2002). La interacción epibionte-basibionte en crustáceos ha sido documentada desde el siglo XIX, sin embargo, en las últimas décadas es que se comienza a caracterizar sus implicaciones fisiológicas, evolutivas y eclógicas (Fernández-Leborans, 2010).

Invertebrados marinos, como crustáceos, moluscos y equinodermos, utilizan materiales diversos en la decoración, entre los más frecuentes se encuentran conchas, guijarros y algas. Al parecer, este comportamiento constituye una estrategia como resultado de la evolución convergente (Wicksten, 1992). La decoración es una de las cinco conductas empleadas por los 
animales para mejorar y modificar el camuflaje de sus propios cuerpos (Stevens y Ruxton, 2018). Los cangrejos emplean el camuflaje para modificar su apariencia y de esta manera se ocultan de sus depredadores (Guzmán, 1979; Cruz-Rivera, 2001; Stevens y Ruxton, 2018).

Las algas epizoicas de la flora de Cuba han sido poco abordadas en las investigaciones. La riqueza de especies sobre animales varía entre siete y 47 (Suárez et al., 2015; Reyes, 2016). Los basibiontes estudiados son el braquiuro Menippe mercenaria (Say, 1818), (Ros y Suárez, 1980) y el gasterópodo Lobatus gigas (Linnaeus, 1758), (Reyes, 2016).

\section{OBJETIVO}

-Identificar por primera vez para las Antillas Mayores las algas epibióticas en tres braquiuros de las familias Epialtidae, Mithracidae y Xanthidae.

\section{MATERIALES Y MÉTODOS}

Área de estudio. Las dos localidades de muestreo están localizadas en las cercanías del Campismo La Mula, en el municipio de Guama, ubicadas a $115 \mathrm{~km}$ al oeste de Santiago de Cuba. Las pocetas intermareales seleccionadas fueron las de Guaimaral, para localización y descripción consultar Cabrera et al. (2017) y la de La Ceiba (coordenadas: 19.945048 N-76.746812 W). Esta ultima ubicada a $2.3 \mathrm{~km}$ al este de la desembocadura del río Turquino y se caracteriza por poseer una profundidad media de $60 \mathrm{~cm}$ y un fondo rocoso dominado por lechos de macroalgas sobre rocas sedimentarias carbonatadas.

Muestreo. Durante junio de 2018, se realizaron tres muestreos en cada poceta durante la mañana para localizar los cangrejos camuflados con algas según la propuesta de Guzmán (1979). La recolecta se realizó mediante búsqueda intensiva durante dos horas mediante buceo SCUBA. Los ejemplares se depositaron en frascos de cristal con agua de mar y se etiquetaron para su posterior análisis en el laboratorio.

Procesamiento de las muestras. Los epibiontes fueron separados del exoesqueleto de los braquiuros mediante el raspado con un bisturí y situados en frascos con una solución de etanol al 70\% (Guzmán, 1979). La identificación taxonómica se realizó basada en caracteres morfológicos, usando literatura especializada y guías de identificación, para los crustáceos (Abele y Kim, 1986; Diez y Jover, 2015; Diez y Espinosa, 2018) y para las algas (Dawes y Mathieson, 2008; Littler et al., 2008). Para la legitimación de los nombres y el ordenamiento taxonómico se siguieron los criterios de WoRMS (2018) y Algabase (Guiry y Guiry, 2018) para los crustáceos y algas respectivamente. Todos los cangrejos se depositaron en las colecciones del Museo Historia Natural "Charles Ramsden de la Torre" y las algas en la Ficoteca Cubana y Antillana, ambos de la Universidad de Oriente.

\section{RESULTADOS}

En las pocetas intermareales de Guaimaral y La Ceiba se recolectaron seis braquiuros camuflados con algas, pertenecientes a tres especies, de las superfamilias Majoidea (2) y Xanthoidea (1). Sobre el exoesqueleto de estos especímenes se encontraron 15 macroalgas epibiontes, ocho rodofíceas, tres feofíceas y cuatro clorofíceas (Tabla I). Las familias Ceramiaceae y Cladophoraceae fueron las de mayor riqueza, con tres especies cada una. 
Tabla I. Lista sistemática de las macroalgas epizoicas identificadas en los exoesqueletos de braquiuros camuflados en pocetas intermareales de la costa suroriental de Cuba.

\section{PHYLUM RHODOPHYTA}

Familia Hapalidiaceae

1. Mesophyllum floridanum (Foslie) W. H. Adey ex M. J. Wynne

2. Jania adhaerens J. V. Lamouroux

Familia Ceramiaceae

3. Ceramium cimbricum H. E. Petersen

4. Ceramium sp.

5. Gayliella transversalis (Collins \& Hervey) T. O. Cho \& Fredericq

Familia Spyridiaceae

6. Spyridia filamentosa (Wulfen) Harvey

Familia Rhizophyllidaceae

7. Ochtodes secundiramea (Montagne) M. Howe

Familia Gracilariaceae

8. Gracilaria sp.

\section{PHYLUM OCHROPHYTA}

Familia Sphacelariaceae

9. Sphacelaria tribuloides Menghini

Familia Acinetosporaceae

10. Feldmannia indica (Sonder) Womersley \& A. Bailey

11. Feldmannia mitchelliae (Harvey) H.-S. Kim

\section{PHYLUM CHLOROPHYTA}

Familia Cladophoraceae

12. Chaetomorpha aerea (Dillwyn) Kützing

13. Cladophora catenata (Linnaeus) Kützing

14. Rhizoclonium riparium (Roth) Kützing ex Harvey

Familia Dasycladaceae

15. Batophora oerstedii J. Agardh 


\section{Lista taxonómica de los braquiuros recolectados}

Orden Decapoda Latreille, 1802

Suborden Pleocynemata Burkenroad, 1963

Infraorden Brachyura Latreille, 1802

Sección Eubrachyura Saint Laurent, 1980

Subsección Heterotremata Guinot, 1977

Superfamilia Majoidea Samouelle, 1819

Familia Epialtidae MacLeay, 1838

Subfamilia Pisinae Dana, 1851

Género Macrocoeloma Miers, 1879.

Macrocoeloma trispinosum (Latreille, 1825). Fig. 1 A-B.

Material examinado. Tres especímenes machos recolectados en la poceta de Guaimaral.

Hábitat. Las muestras fueron tomadas en fondos duros, con rocas sueltas cubiertas por algas y en fondos arenosos con Thalassia testudinum Banks ex König. Se recolectaron a $35 \mathrm{~cm}$ de profundidad, se han reportado desde el mesolitoral hasta los $82 \mathrm{~m}$.

Epibiontes. Tres rodofíceas: Ceramium cimbricum, Ceramium sp. y Gracilaria sp. Dos feofíceas: Sphacelaria tribuloides y Feldmannia indica. Tres clorofíceas: Chaetomorpha aerea, Cladophora catenata y Batophora oerstedii.

Familia Mithracidae MacLeay, 1838

Género Omalacantha Streets, 1871.

Omalacantha bicornuta (Latreille, 1825). Fig. 1 C-D.

Material examinado. Dos especímenes, uno macho y otro hembra, recolectados en La Ceiba.

Hábitat. En mantos de macroalgas dominados por Laurencia, Gracilaria y Digenia, creciendo sobre rocas sedimentarias. Se recolectaron a $40 \mathrm{~cm}$ de profundidad, se han registrado hasta los $70 \mathrm{~m}$.

Epibiontes. Cinco rodofíceas: C. cimbricum, Gayliella transversalis, Spyridia filamentosa, Octhodes secundiramea y Gracilaria sp. Dos feofíceas: S. tribuloides y Feldmannia mitchellidae. Dos clorofíceas: C. catenata y Rhizoclonium riparium.

Superfamilia Xanthoidea Dana, 1851

Familia Xanthidae Dana, 1851

Subfamilia Acteinae Alcock, 1898

Género Actaea De Haan, 1833.

Actaea bifrons Rathbun, 1898. Fig. 1 E-F.

Material examinado. Dos especímenes machos recolectados en Guaimaral.

Hábitat. Las muestras fueron tomadas en fondos arenosos con Thalassia testudinum Banks ex König y macroalgas asociadas. Se recolectaron a $30 \mathrm{~cm}$ de profundidad, se ha registrado su presencia hasta los $72 \mathrm{~m}$.

Epibiontes. Dos rodofíceas: Mesophyllum floridanum y Jania adhaerens. Una feofícea: $S$. tribuloides. Cuatro clorofíceas: $C$. catenata, Chaetomorpha aerea, $R$. riparium y B. oerstedii. 


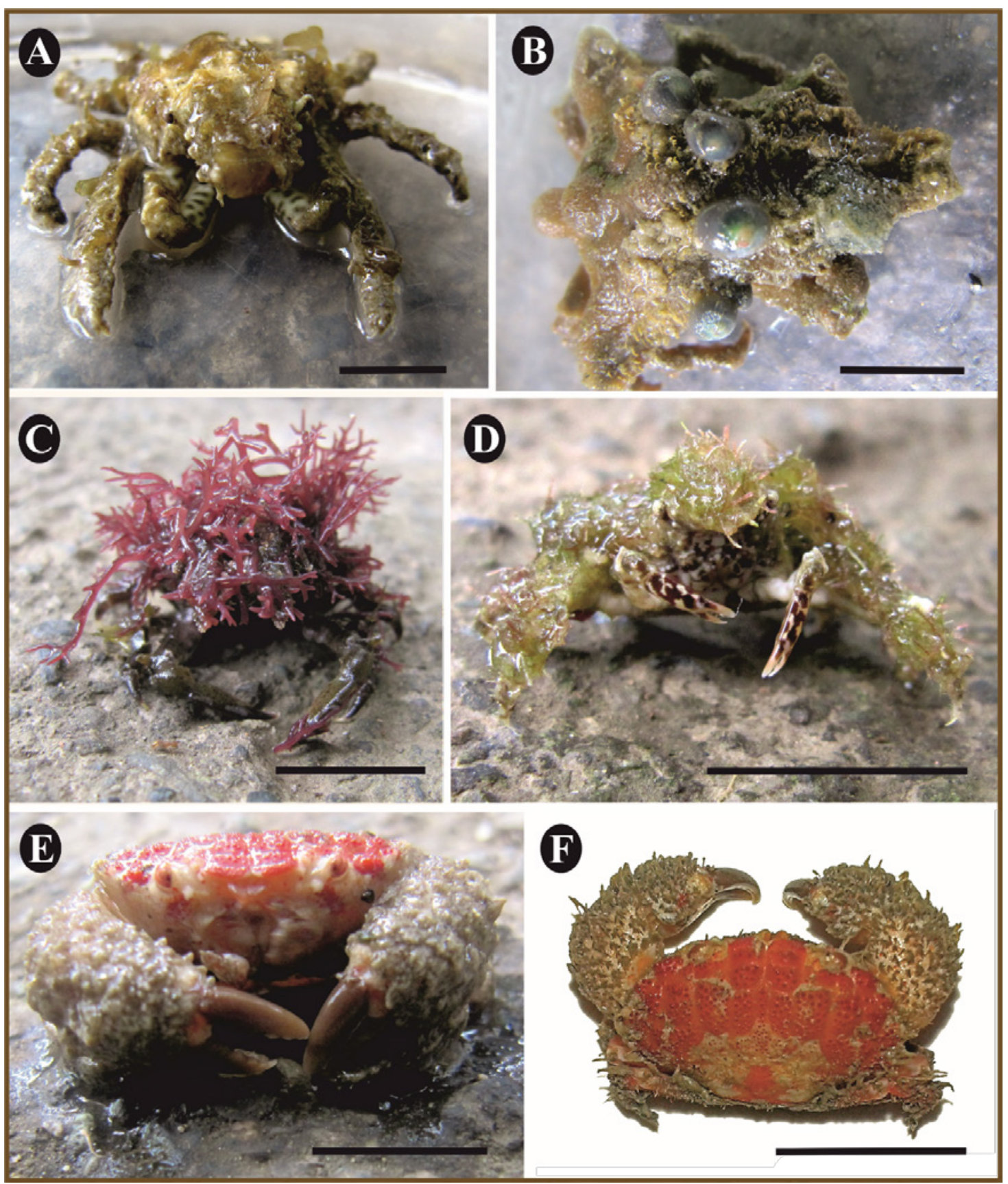

Figura 1. Crustáceos camuflados con algas epibiontes en pocetas intermareales de la costa suroriental de Cuba. A-B: Macrocoeloma trispinosum. C-D: Omalacantha bicornuta. E-F: Actaea bifrons. Escala=1 cm. 
Las algas epibiontes presentaron variaciones en cuanto a la riqueza y distribución entre los especímenes de braquiuros enmascarados recolectados en las pocetas intermareales de Guaimaral y La Ceiba (Fig. 2). La mayor riqueza de algas epibióticas se consignó en $O$. bicornuta y además fue el braquiuro con mayor número de macroalgas de distribución restringidas en su exoesqueleto. Las macroalgas Sphacelaria tribuloides y Cladophora catenata fueron registradas en todas las especies de braquiuros camuflados.

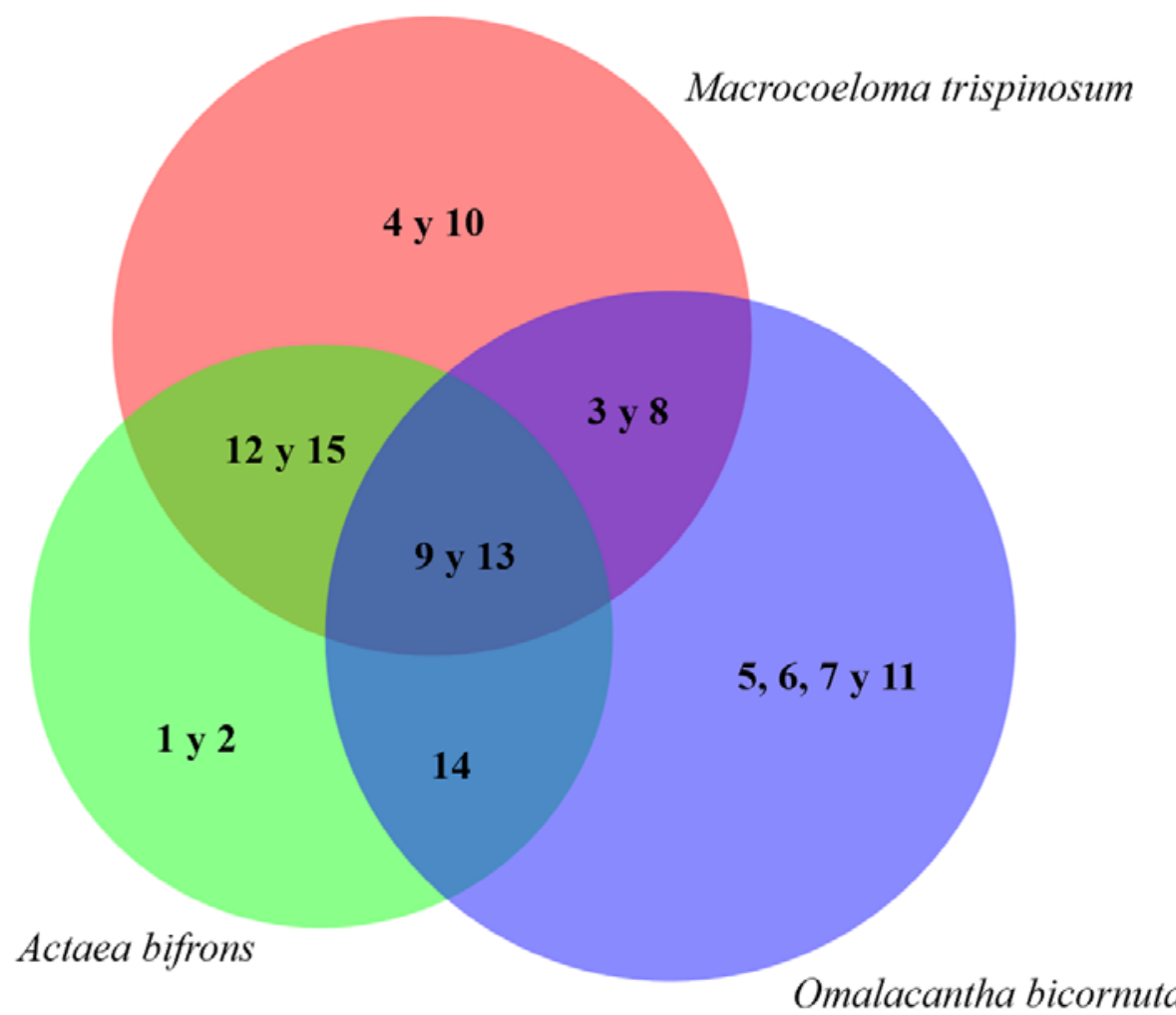

Figura 2. Distribución de las algas epibióticas de cangrejos camuflados en pocetas intermareales de la costa suroriental de Cuba.

\section{DISCUSIÓN}

Los cangrejos enmacarados presentes en las pocetas intermareales de Guimaral y la Ceiba son especies abundantes y frecuentes en comunidades marinas con numerosos microhábitas, como resultado de la presencia de rocas, angiospermas marinas y macroalgas (González-Gómez et al., 2018). El hábitat y la distribución batimétrica se corresponde con otros trabajos realizados en Cuba (Diez y Jover, 2015; Diez y Espinosa, 2018), el mar Caribe (CarmonaSuárez y Poupin, 2016) y el Golfo de México (Felder et al., 2009). En estos ecosistemas utilizan las algas para enmascararse y en la alimentación. 
Los géneros de algas consignadas sobre los exoesqueletos de los cangrejos camuflados en Guaimaral y La Ceiba han sido consignados como epibióticas en crustáceos, moluscos y vertebrados marinos (Ros y Suárez, 1980; Reyes, 2016; Violante-Huerta, 2018). Las 15 especies constituyen nuevas adiciones a la lista de algas epibióticas sobre crustáceos de las Antillas Mayores. Fenómeno que se debe a la carencia de investigaciones encaminadas a caracterizar las relaciones entre macroalgas que crecen sobre braquiuros, solo se registra el realizado por Ros y Suarez (1980), en el que se adicionan dos especies. Es de destacar que hoy es tema de debate científico las relaciones conductuales del enmascaramiento en crustáceos marinos y el papel que desempeñan en la selección del hábitat, elección de la pareja y en la subsistencia cuando los alimentos son escasos (Wicksten, 1992; Fernández-Leborans, 2010; Gonzaléz-Gómez et al., 2018; Stevens y Ruxton, 2018).

La mayor riqueza de algas epibióticas pertenecientes a Ceramiaceae y Cladophoraceae corresponde con el patrón observado para el molusco L. gigas (Reyes, 2016), el crustáceo O. bicornutus (Guzmán, 1979) y en tortugas marinas (Loza, 2011). Es de destacar que estas familias son identificadas como las de mayor riqueza en estos hábitats por sus características de ser algas filamentosas con estructuras de fijación que permite adherirse a diversos sustratos (Jover et al., 2012). La mayor riqueza de algas filamentosas y de pequeño tamaño ha sido atribuido como especies colonizadoras primarias sobre sustratos vivos como las tortugas marinas y los taxa de mayor riqueza se corresponden a Ceramiaceae, Cladophoraceae y el complejo de algas filamentosas pardas donde se encuentra Ectocarpus (Loza, 2011).

La mayor riqueza de algas epibiontes en los crustáceos de la superfamilia Majoidea se debe a las características del exoesqueleto, que posee una mayor ornamentación en su superficie y de esta manera favorece la colonización (Wicksten, 1992; Fernandez-Leborans, 2010). Además, en estas especies se ha demostrado la utilización de los quelípedos para tomar algas del sustrato e incorporarlas a su camuflaje (Guzmán, 1979).

Las diferencias en la distribución de las algas epibióticas en las tres especies estudiadas pudo deberse a la disponibilidad de macroalgas dominantes y a las características del sustrato de los microhábitats (Wicksten, 1992; Fernández-Leborans, 2010). Otro factor interesante, no evaluado en la investigación, es la talla de los basibiontes, pues es manifiesta la presencia de decorados diferentes entre juveniles y adultos (Guzmán, 1979). Además, no se descarta la presencia de especificidad en la selección del enmascaramiento. Este fenómeno ha sido identificado en cangrejos camuflados en el Mediterráneo (Cruz-Rivera, 2001). Por lo que esta investigación, mas que respuestas al fenómeno de la epibiosis en crustáceos litorales, abre múltiples interrogantes para explicar este comportamiento.

\section{CONCLUSIÓN}

En los exoesqueletos de los braquiuros Macrocoeloma trispinosum, Omalacantha bicornuta y Actaea bifrons se identificaron 15 macroalgas epibionticas. Las especies de epibiontes mejor distribuidas entre los tres braquiuros se caracterizaron por poseer un talo filamentoso y de pequeño tamaño. La mayor riqueza de algas se observó en el cangrejo decorador O. bicornuta. Mientras que las algas Sphacelaria tribuloides y Cladophora catenate se identificaron en todos los individuos de cangrejos enmascarados recolectados. 


\section{AGRADECIMIENTOS}

A la Universidad de Oriente, y en especial al proyecto "Biodiversidad de Cuba oriental" por las facilidades logísticas que ayudaron a la realización de la investigación. A Alejandro Catalá por su acertada ayuda en la selección y confección de las figuras y a Yander L. Diez por la revisión taxonómica de los crustáceos. A todos los colegas que revisaron el manuscrito y brindaron sus aportes y sugerencias.

\section{LITERATURA CITADA}

Abele, L. G. y W. Kim. 1986. An illustrated guide to the marine decapod crustaceans of Florida. Florida, USA: Florida State University, 209 pp.

Cabrera, A., R. Muñoz, C. Batista, F. Álvarez y A. Jover. 2017. Relaciones tróficas entre equinoideos (Echinodermata) de las pocetas intermareales de La Sardina y Guaimaral, costa suroriental de Cuba. Novitates Caribaea, 11: 51-68.

Carmona-Suárez, C. y J. Poupin. 2016. Majoidea crabs from Guadeloupe Island, with a documented list of species for the Lesser Antilles (Crustacea, Decapoda, Brachyura, Majoidea). Zoosystema, 38 (3): 353-387.

Cruz-Rivera, E. 2001. Generality and specificity in the feeding and decoration preferences of three Mediterranean crabs. Journal of Experimental Marine Biology and Ecology, 266: 17-31.

Dawes, C. y A. Mathieson. 2008. The Saeweeds of Florida. University of Florida Press, 591 pp.

Diez, Y. L. y A. Jover. 2015. List ofmarine crabs (Decapoda: Anomura and Brachyura) of shallow littoral of Santiago de Cuba, Cuba. Check List, 11 (2): 1601.

Diez, Y. L. y J. Espinosa. 2018. New data on the distribution of crabs (Decapoda: Anomura and Brachyura) in Cuba. Revista de Investigaciones Marinas, 38 (1): 126-145.

Felder, D. L., F. Álvarez, J. W. Goy y R. Lemaitre. 2009. Decapoda (Crustacea) of the Gulf of Mexico, with comments on the Amphionidacea, pp. 1019-1104 in Felder, D. L. and D. K. Camp (Eds.), Gulf of Mexico-Origins, Waters, and Biota. Biodiversity. Texas A\&M University Press, College Station, Texas.

Fernández-Leborans, G. 2010. Epibiosis in Crustacea: an overview. Crustaceana, 83 (5): 549-640.

González-Gómez, R., P. Briones-Fourzán, L. Álvarez-Filip y E. Lozano-Álvarez. 2018. Diversity and abundance of conspicuous macrocrustaceans on coral reefs differingin level of degradation. PeerJ, 6: e4922; DOI 10.7717/peerj.4922.

Guiry, M. y G. Guiry. 2018. Algabase. Disponible: www.algabase.org. Accedido: 6 de octubre de 2018.

Guzmán, H. 1979. Comportamiento decorador con algas en el cangrejo Microphyrys bicornutus Latreille (Majidae; Decapoda). Revista de Biología Tropical, 27 (2): 321-327. 
Jover, A., L. Reyes, L. Gómez y A. Suárez. 2012. Variación espacial y temporal de las macroalgas del mesolitoral rocoso en Aguadores-Baconao, Cuba I: Composición. Revista de Investigaciones Marinas, 32: 38-49.

Littler, D., M. Littler y M. Dennis. 2008. Submersed Plants of the Indian River Logoon. Florida, Offshore Graphics, Inc., 548 pp.

Loza, A. L. 2011. Ecosistemas errantes: epibiontes como indicadores biogeográficosde tortugas marinas de Canarias. Tesis de Doctorado, Universidad de Las Palmas de Gran Canaria, España, 362 pp.

Reyes, L. M. 2016. Macroalgas epizoicas en Lobatus gigas en el Parque Nacional "Jardines de la Reina”, Camagüey, Cuba. Hombre, Ciencia y Tecnología, 20 (2): 67-74.

Ros, R. M. y A. M. Suárez. 1980. Epibiosis en el cangrejo moro Menippe mercenaria (Say, 1818). Revista de Investigaciones Marinas, 1 (1): 5-17.

Schämer, M. T. 2005. A Survey of the Epibiota of Hawksbill Sea Turtle (Eretmochelys imbricata) of Mona Island, Puerto Rico. Tesis de Maestría. Universidad de Puerto Rico, Mayagüez, $82 \mathrm{pp}$.

Stevens, M. y G. Ruxton. 2018. The key role of behavior in animal camouflage. Biological Reviews, in press, DOI: 10.1111/brv.12438.

Suárez, A. M., B. Martínez-Daranas y Y. Alfonso. 2015. Macroalgas marinas de Cuba. Editorial UH, La Habana, Cuba, 246 pp.

Taylor, P. y M. Wilson. 2002. A new terminology for marine organisms inhabiting hard substrates. PALAIOS, 17: 522-525.

Violante-Huerta, M. 2018. La epibiosis en los grandes vertebrados marinos de México: una revisión y su relevancia ecosistémica. Revista Peruana de Biología, 25 (3): 335-342; DOI: $10.15381 /$ rpb.v25i3.14786.

Wicksten, M. 1992. A Review and a Model of Decorating Behavior in Spider Crabs (Decapoda, Brachyura, Majidae). Crustaceana, 64 (3): 314-325.

WoRMS. 2018. World Register of Marine Species. Disponible: www.marinespecies.org. Accedido: 20 de octubre de 2018. DOI: 10.14284/170.

[Recibido: 08 de noviembre, 2018. Aceptado para publicación: 12 de diciembre, 2018] 